\title{
Prognostic grading system specifically for elderly patients with brain metastases after stereotactic radiosurgery: a 2-institution study
}

\author{
*Masaaki Yamamoto, MD, PhD, ${ }^{1,2}$ Toru Serizawa, MD, PhD, ${ }^{3,4}$ Yoshinori Higuchi, MD, PhD, ${ }^{4}$ \\ Osamu Nagano, MD, PhD, ${ }^{5}$ Hitoshi Aiyama, MD, ${ }^{1,6}$ Takao Koiso, MD, PhD, ${ }^{1,6}$ \\ Shinya Watanabe, MD, PhD, ${ }^{1,7}$ Takuya Kawabe, MD, PhD, ${ }^{8}$ Yasunori Sato, $\mathrm{PhD},{ }^{9}$ and \\ Hidetoshi Kasuya, MD, PhD²
}

\begin{abstract}
${ }^{1}$ Katsuta Hospital Mito GammaHouse, Hitachi-naka, Ibaraki; ${ }^{2}$ Department of Neurosurgery, Tokyo Women's Medical University Medical Center East, Tokyo; ${ }^{3}$ Tokyo Gamma Unit Center, Tsukiji Neurological Clinic, Tokyo; ${ }^{4}$ Department of Neurological Surgery, Chiba University Graduate School of Medicine, Chiba; ${ }^{5}$ Gamma Knife House, Chiba Cerebral and Cardiovascular Center, Ichihara; ${ }^{6}$ Department of Neurosurgery, Faculty of Medicine, and ${ }^{7}$ Tsukuba Clinical Research and Development Organization, University of Tsukuba; ${ }^{8}$ Department of Neurosurgery, Kyoto Prefectural University of Medicine Graduate School of Medical Sciences, Kyoto; and 'Department of Clinical Research Center, Chiba University Graduate School of Medicine, Chiba, Japan
\end{abstract}

OBJECTIVE With the aging of the population, increasing numbers of elderly patients with brain metastasis (BM) are undergoing stereotactic radiosurgery (SRS). Among recently reported prognostic grading indexes, only the basic score for brain metastases (BSBM) is applicable to patients 65 years or older. However, the major weakness of this system is that no BM-related factors are graded. This prompted the authors to develop a new grading system, the elderly-specific (ES)-BSBM.

METHODS For this IRB-approved, retrospective cohort study, the authors used their prospectively accumulated database comprising 3267 consecutive patients undergoing Gamma Knife SRS for BMs during the 1998-2016 period at the Mito GammaHouse. Among these 3267 patients, 1789 patients $\geq 65$ years of age were studied (Yamamoto series [Yseries]). Another series of 1785 patients $\geq 65$ years of age in whom Serizawa and colleagues performed Gamma Knife SRS during the same period (Serizawa series [S-series]) was used for validity testing of the ES-BSBM.

RESULTS Two factors were identified as strongly impacting longer survival after SRS by means of multivariable analysis using the Cox proportional hazard model with a stepwise selection procedure. These factors are the number of tumors (solitary vs multiple: HR 1.450,95\% Cl 1.299-1.621; $p<0.0001)$ and cumulative tumor volume $\left(\leq 15 \mathrm{~cm}^{3} \mathrm{vs}>\right.$ $\left.15 \mathrm{~cm}^{3}: \mathrm{HR} 1.311,95 \% \mathrm{Cl} 1.078-1.593 ; p=0.0067\right)$. The new index is the addition of scores 0 and 1 for these 2 factors to the BSBM. The ES-BSBM system is based on categorization into 3 classes by adding these 2 scores to those of the original BSBM. Each ES-BSBM category has 2 possible scores. For the category ES-BSBM 4-5, the score is either 4 or 5; for ES-BSBM 2-3, the score is either 2 or 3; and for ES-BSBM $0-1$, the score is either 0 or 1 . In the Y-series, the median survival times (MSTs, months) after SRS were 17.5 (95\% Cl 15.4-19.3) in ES-BSBM 4-5, $6.9(95 \% \mathrm{Cl} 6.4-7.4)$ in ES-BSBM 2-3, and $2.8(95 \% \mathrm{Cl} 2.5-3.6)$ in ES-BSBM $0-1$ ( $p<0.0001)$. Also, in the S-series, MSTs were, respectively, 20.4 (95\% Cl 17.2-23.4), 7.9 (95\% Cl 7.4-8.5), and 3.2 (95\% Cl 2.8-3.6) ( $<$ <0.0001). The ES-BSBM system was shown to be applicable to patients with all primary tumor types as well as to those 80 years or older.

CONCLUSIONS The authors found that the addition of the number of tumors and cumulative tumor volume as scoring factors to the BSBM system significantly improved the prognostic value of this index. The present study is strengthened by testing the ES-BSBM in a different patient group.

https://thejns.org/doi/abs/10.3171/2018.7.GKS181458

KEYWORDS brain metastases; radiation therapy; stereotactic radiosurgery; Gamma Knife; elderly patients; prognostic index; oncology

ABBREVIATIONS BM = brain metastasis; BSBM = basic score for brain metastases; ES-BSBM = elderly-specific BSBM; GI = gastrointestinal; GKSRS = Gamma Knife SRS; KPS = Karnofsky Performance Status; MST = median survival time; NSCLC = non-small cell lung cancer; RPA = recursive partitioning analysis; S-series = Serizawa series; SCLC = small cell lung cancer; SRS = stereotactic radiosurgery; WBRT = whole-brain radiotherapy; Y-series = Yamamoto series.

SUBMITTED May 24, 2018. ACCEPTED July 25, 2018.

INCLUDE WHEN CITING DOI: 10.3171/2018.7.GKS181458.

${ }^{*}$ M.Y. and T.S. contributed equally to this study. 
$\mathrm{A}$ $\mathrm{S}$ the population of older individuals continues to increase, clinicians can anticipate treating more elderly cancer patients. ${ }^{28}$ Even in elderly patients with systemic cancer, prolonged survival can now be achieved with the application of recently developed diagnostic technologies and more effective treatment options..$^{10,21}$ The longer cancer patients survive, the more frequently physicians encounter brain metastases (BMs). Therefore, management of elderly BM patients has now become one of the main concerns for physicians who provide cancer treatment. ${ }^{1,6,8,12,14,15,21}$ Watanabe et al. reported that increasing numbers of elderly BM patients have been treated with stereotactic radiosurgery (SRS) and found that treatment results, even those in patients 80 years or older, were not unfavorable. ${ }^{21}$ Very recently, Chen et al. reported that 811 BM patients older than 70 years who underwent wholebrain radiotherapy (WBRT), historically a gold standard for managing BM patients, experienced increased toxicity compared with SRS in elderly and very elderly ( $>80$ years) patients with BMs. ${ }^{1}$

Because many factors affect the outcomes of patients with BMs, a good grading index facilitates treatment selection. However, to the best of our knowledge, there are no prognostic indexes specific to elderly BM patients. Although the recursive partitioning analysis (RPA) system proposed by Gaspar et al. is widely used, patients 65 years or older are initially excluded from class $1 .^{2}$ In our modified RPA system, class $1+2 \mathrm{a}$ includes patient age 65 years or older. ${ }^{25,26}$ However, this modified RPA system was derived fundamentally from the RPA system and, therefore, is not considered to be adequately applicable to elderly patients. After publication of the RPA system, the following 3 indexes were proposed: 1) Score Index for Radiosurgery, the sum of scores (0-2) for each of the 5 prognostic factors included (age, Karnofsky Performance Status [KPS] score, systemic disease status, number of lesions, and volume of the largest lesion); ${ }^{22}$ 2) basic score for brain metastases (BSBM), the sum of scores (0 and 1) for the 3 prognostic factors included (KPS score, controlled primary tumor, and extracranial metastases); ${ }^{9}$ and 3, Graded Prognostic Assessment, the sum of scores $(0,0.5$, or 1.0$)$ for each of the 4 prognostic factors included (age, KPS score, number of lesions, and extracranial metastases). ${ }^{17}$ Among these 3 indexes, only BSBM shows good applicability to elderly patients, despite age not being included as a scoring factor. However, the BSBM has a crucial weakness in that the system includes no factors relating to the actual development of a BM. This prompted us to modify the BSBM system, by adding 2 factors, tumor number and cumulative tumor volume, to the original BSBM system. We call this modification the elderly-specific (ES)-BSBM. The addition of these 2 factors to the BSBM index significantly improved its prognostic value for patients of advanced age.

This study had 2 parts. First, using clinical factor median survival time (MST) analysis of 1789 BM patients 65 years or older who underwent Gamma Knife SRS (GKSRS) performed by the first author (M.Y.), we developed the ES-BSBM. Subsequently, we tested the validity of this index using another series, comprising 1785 patients independently undergoing GKSRS performed by the second author (T.S.). In each series, 1 author (either M.Y. or T.S.) was responsible for all aspects of patient selection, dose planning, dose selection, performing GKSRS, and collecting follow-up data.

\section{Methods}

\section{Patient Population}

For this IRB-approved, retrospective cohort study, we used our prospectively accumulated database including 3267 consecutive patients undergoing GKSRS for BMs during the 1998-2016 period at the Mito GammaHouse. Among these 3267 patients, 1789 patients who were 65 years or older were studied (Yamamoto [Y]-series). A different series of 1785 patients who were 65 years or older and had been treated by the second author (T.S.) and his colleagues using GKSRS during the same period (Serizawa [S]-series) was used for validity testing of our ESBSBM.

Table 1 summarizes clinical characteristics of the patient groups in the 2 series. The patients had all been referred to us by other facilities to undergo SRS. In some cases, the primary physician had determined the indications for surgery and/or radiotherapy prior to SRS. Therefore, before undergoing SRS, 279 (16\%) of 1786 patients in the Y-series and 203 (11\%) of the 1785 S-series had undergone surgical BM removal, while WBRT had been administered to $70(4 \%)$ and $106(6 \%)$ patients, respectively.

The treatment strategy was explained in detail prior to SRS to each patient and at least one adult relative by either the first (M.Y.) or the second (T.S.) author. Written informed consent was obtained from all patients. We have described our radiosurgical techniques in detail in earlier publications. ${ }^{24,25}$ In brief, standard single-session GKSRS was performed using a Leksell Gamma Knife Model B or $\mathrm{C}$ unit and, more recently, the Leksell Gamma Knife Perfexion (Elekta Instruments AB), in both series. However, most patients whose largest tumor volume exceeded $10 \mathrm{~cm}^{3}$ received 3-stage GKSRS. ${ }^{4}$

After SRS, the referring physicians carried out routine management in all cases. The patients were recommended to receive clinical and neuroimaging examinations at an interval of approximately 2-3 months.

\section{Statistical Analysis}

The intention-to-treat principle was applied to all analyses of the data. For the analyses involving baseline variables, we constructed summary statistics using frequencies and proportions for the categorical data and medians, ranges, and IQRs for the continuous variables. Comparisons of patient characteristics were carried out using the Fisher exact test for categorical outcomes and t-tests for continuous variables. The Kaplan-Meier method was applied to determine overall survival.

All comparisons were planned, and the statistical tests were 2-sided; $\mathrm{p}<0.05$ indicated statistical significance. All statistical analyses were performed by one of the authors (Y.S.) using SAS software (version 9.3, SAS Institute) and the R statistical program, version 3.0.0. Before the statistical analyses, the database was cleaned of data and typographical errors (by Y.H.). These 2 authors were not involved in either SRS treatment or patient follow-up. 


\section{Results}

\section{Survival and Pre-SRS Clinical Factors Impacting Longer Survival}

Although clinical characteristics differed between the 2 series (Table 1), post-SRS MSTs were very similar, 7.5 (95\% CI 7.1-8.0) months in the Y-series and 7.8 (95\% CI 7.4-8.4) months in the S-series (HR 0.976, 95\% CI 0.9101.046; $p=0.4886$ ), as shown in Fig. 1 .

\section{ES-BSBM}

The clinical factors that showed statistical significance for survival in the univariable analyses were included in the multivariable analysis (Table 2). Four clinical factors were found to significantly impact longer survival: female sex, solitary tumor, cumulative tumor volume smaller than $15 \mathrm{~cm}^{3}$, and prior surgery. Although the longer survival of female than male BM patients is widely known, sex was not included as a clinical factor in the previously reported prognostic indexes. ${ }^{2,9,17,22,25,26}$ Usually, the proportion of patients who have had prior surgery is rather small. ${ }^{16,23,27}$ Therefore, excluding these 2 factors, our new index is the sum of scores ( 1 and 0 ) for the other 2 additional factors, i.e., solitary versus multiple BMs and cumulative tumor volume $15 \mathrm{~cm}^{3}$ or larger vs smaller than $15 \mathrm{~cm}^{3}$ (Table 3 ). Also, multivariable analysis using the S-series database demonstrated that these 2 factors significantly impact longer survival; i.e., solitary tumor (HR 1.456, 95\% CI 1.3041.628; $\mathrm{p}<0.0001)$ and cumulative tumor volume smaller than $15 \mathrm{~cm}^{3}$ (HR 1.672, 95\% CI 1.465-1.902; p < 0.0001) favor longer survival. The ES-BSBM system is based on categorization into 3 classes by adding these 2 scores to those of the original BSBM. Each ES-BSBM category has 2 possible scores. For the ES-BSBM category of 4-5, the score is either 4 or 5; for ES-BSBM 2-3, the score is either 2 or 3 ; and for ES-BSBM $0-1$, the score is either 0 or 1 .

\section{Survival Analyses Using ES-BSBM}

Figure 2A shows the Kaplan-Meier plots for the 3 subclasses of the Y-series. The MSTs differed significantly, with no overlapping of $95 \%$ CIs among the 3 classes. This ES-BSBM was applied to the S-series as a validity test to determine its applicability to other BM patient groups. As shown in Fig. 2B (S-series), the MSTs also differed significantly, again with no overlapping of $95 \%$ CIs among the 3 classes. Also, based on all patients in both series, this new index was confirmed to be applicable to original tumor categories, i.e., non-small cell lung cancer (NSCLC; Fig. 3A), small cell lung cancer (SCLC; Fig. 3B), gastrointestinal (GI) tract cancer (Fig. 3C), breast cancer (Fig. 3D), kidney cancer (Fig. 3E), and others (Fig. 3F). Furthermore, based on all patients in the 2 series combined, this new index was confirmed to be applicable to 2 patient age groups: those 65-79 years and 80 years or older (Fig. 4).

\section{Discussion}

We consider this new prognostic index, ES-BSBM, to be the first prognostic grading system developed especially for elderly patients with BMs. The present study clearly demonstrated the ES-BSBM to be applicable to the Y-
TABLE 1. Summary of demographic characteristics in 1789 Yamamoto-series and 1785 Serizawa-series patients

\begin{tabular}{|c|c|c|c|}
\hline Characteristics & Yamamoto Series & Serizawa Series & $p$ Value \\
\hline No. of patients & 1789 & 1785 & \\
\hline Age in yrs & & & 0.2629 \\
\hline Median (IQR) & $73(68-77)$ & $73(68-76)$ & \\
\hline Maximum & 96 & 96 & \\
\hline Sex & & & 0.0550 \\
\hline Male & $1176(66)$ & $1118(63)$ & \\
\hline Female & $613(34)$ & $667(37)$ & \\
\hline $\begin{array}{l}\text { Neurological } \\
\text { symptom(s) }\end{array}$ & & & 0.1156 \\
\hline Yes & $949(53)$ & $899(50)$ & \\
\hline No & 840 (47) & $886(50)$ & \\
\hline KPS score & & & 0.2768 \\
\hline$\geq 80 \%$ & $1314(73)$ & $1282(72)$ & \\
\hline$\leq 70 \%$ & $475(27)$ & $503(28)$ & \\
\hline BSBM & & & $<0.0001$ \\
\hline 3 & $274(17)$ & $174(10)$ & \\
\hline 2 & $688(39)$ & $604(34)$ & \\
\hline 1 & $632(35)$ & $722(40)$ & \\
\hline 0 & $195(11)$ & $285(28)$ & \\
\hline Primary cancer & & & 0.0968 \\
\hline NSCLC & $1049(60)$ & $1076(60)$ & \\
\hline SCLC & $222(12)$ & $189(11)$ & \\
\hline GI tract & $221(12)$ & $246(14)$ & \\
\hline Breast & $86(5)$ & $101(6)$ & \\
\hline Kidney & $69(4)$ & $55(3)$ & \\
\hline Other & $142(8)$ & $118(7)$ & \\
\hline Primary cancer status & & & 0.1731 \\
\hline Controlled & $540(30)$ & $501(28)$ & \\
\hline Not controlled & $1249(70)$ & $1284(72)$ & \\
\hline $\begin{array}{l}\text { Extracranial metas- } \\
\text { tases }\end{array}$ & & & $<0.0001$ \\
\hline Yes & $813(45)$ & $1116(63)$ & \\
\hline No & $976(55)$ & $669(37)$ & \\
\hline Prior surgery & & & 0.0002 \\
\hline Yes & $279(16)$ & $203(11)$ & \\
\hline No & $1510(84)$ & $1582(89)$ & \\
\hline Prior WBRT & & & 0.0053 \\
\hline Yes & $70(4)$ & $106(6)$ & \\
\hline No & $1719(96)$ & $1679(94)$ & \\
\hline No. of tumors & & & 0.5387 \\
\hline Median (IQR) & $3(1-4)$ & $3(1-7)$ & \\
\hline Range & $1-89$ & $1-100$ & \\
\hline \multicolumn{4}{|l|}{ Tumor vol, $\mathrm{cm}^{3}$} \\
\hline Cumulative & & & 0.0010 \\
\hline Median (IQR) & $4.9(1.7-12.0)$ & $4.1(1.3-10.9)$ & \\
\hline Range & $0.01-126.2$ & $0.02-82.9$ & \\
\hline Largest tumor & & & $<0.0001$ \\
\hline Median (IQR) & $3.4(1.1-8.3)$ & $2.6(0.6-7.2)$ & \\
\hline Range & $0.01-94.2$ & $0.01-69.0$ & \\
\hline
\end{tabular}


» CONTINUED FROM PAGE 97

TABLE 1. Summary of demographic characteristics in 1789 Yamamoto-series and 1785 Serizawa-series patients

\begin{tabular}{cccc}
\hline Characteristics & Yamamoto Series & Serizawa Series & $p$ Value \\
\hline Dose (Gy) & & & $<0.0001$ \\
\hline Minimum & & & \\
\hline Median (IQR) & $22.0(20.0-24.0)$ & $20.0(19.0-21.0)$ & \\
\hline Range & $5.8-32.0$ & $8.0-30.0$ & \\
\hline Maximum & & & \\
\hline Median (IQR) & $35.0(30.0-40.0)$ & $36.4(29.4-40.1)$ & \\
\hline Range & $18.3-50.0$ & $16.4-51.3$ & \\
\hline
\end{tabular}

Values are presented as the number of patients unless stated otherwise.

series, and its validity was then tested using the S-series. Several diagnosis-specific grading systems, tailored to the unique features of each primary cancer category, have recently been proposed. ${ }^{13,18-20}$ These systems are not complicated for physicians who manage patients with a single primary cancer category. Furthermore, incorporating molecular subtypes into these recently proposed prognostic grading indexes is considered to be problematic. This is because, while their incorporation is absolutely scientific, the new molecular subtypes have been discovered and come into use over several years, thereby necessitating frequent changes in the grading indexes. The major problem with such prognostic grading indexes is that they are not applicable to retrospective studies. We consider a single prognostic grading system covering patients with all primary cancer types to be much more convenient for physicians who manage patients with various primary tumors. From this viewpoint, our ES-BSBM system is more broadly applicable to BM patients, regardless of their primary cancer types, i.e., NSCLC, SCLC, GI tract cancer, breast cancer, renal tumors, and so on. Furthermore, this system can be applied to nonelderly BM patients. However, as mentioned above, grading indexes have already been established and, therefore, we consider it to be unreasonable to use the ESBSBM for nonelderly BM patients.

Among various clinical factors apparently related to longer survival times, we added 2 factors, i.e., tumor number (solitary vs multiple) and cumulative tumor volume $\left(<15 \mathrm{~cm}^{3}\right.$ vs $\left.\geq 15 \mathrm{~cm}^{3}\right)$ to the original BSBM system. As stated above, multivariable analysis demonstrated the HRs of these 2 factors to be far higher than those of others, except for the 3 factors already incorporated in the original BSBM (Table 2). Our previous studies, both prospective and retrospective, clearly demonstrated that patients with solitary BMs survived significantly longer than those with multiple BMs..$^{16,23,26}$ Also, larger cumulative tumor volumes were shown to be unfavorable prognostic factors, as Marcus et al. ${ }^{11}$ and Serizawa et al. ${ }^{16}$ have reported. Therefore, using 2 aforementioned factors as additional clinical factors for scoring is considered to be justified.

A cross-national comparative study of 7 industrialized countries conducted in 2000 found that the proportions of individuals 65 years or older ranged from $12.6 \%$ to $18.1 \% .^{28}$ It has also been estimated that the proportion of elderly

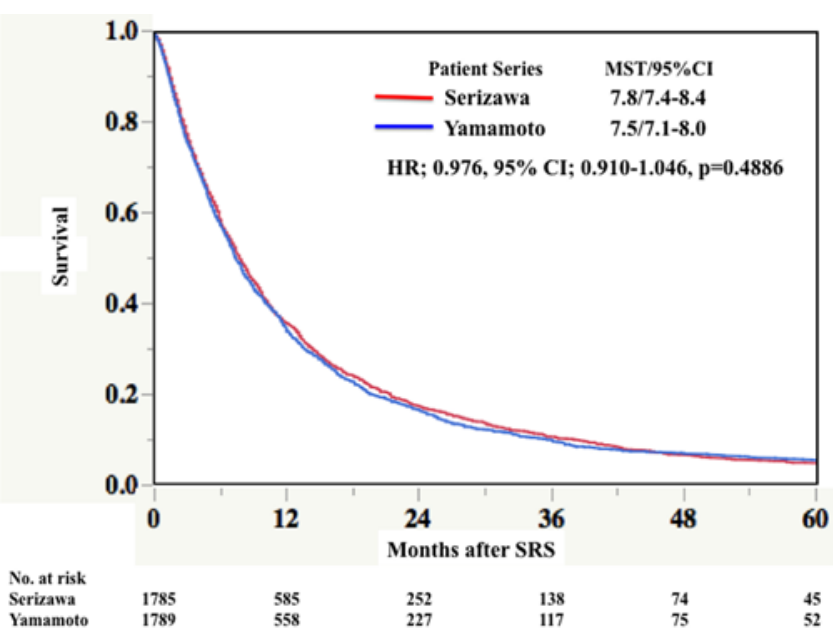

FIG. 1. Kaplan-Meier analysis of overall survival for the 2 series of patients. MST = median survival time (months).

persons will reach $20 \%-28 \%$ of the entire population in the majority of industrialized nations by the year 2030. In Japan particularly, 26.7\% of the population was at least 65 years in 2015, and this number is expected to reach $31.6 \%$ by 2030 (https://www.mhlw.go.jp/english/social_security/ dl/social_security6-g.pdf). Due to the rapidly increasing population of elderly individuals, physicians are now taking a growing interest in the management of elderly cancer patients with BMs. ${ }^{6,8,10,12,14,15}$ Therefore, the development of a more suitable grading index is eagerly awaited. Particularly, clinicians are now faced with the challenge of an increasing number of very elderly $\mathrm{BM}$ patients: those 80 years and older. ${ }^{1,21,29}$ As shown in Fig. 4B, our ES-BSBM was demonstrated to be applicable to patients of advanced age with BM.

In devising the ES-BSBM, based on statistical analyses using Y-series patients, we added the scores for 2 clinical factors (solitary vs multiple and cumulative tumor volume $15 \mathrm{~cm}^{3}$ or larger vs smaller than $15 \mathrm{~cm}^{3}$ ). Several retrospective studies based on more than 1000 BM patients who underwent SRS have shown that patients with just 1 tumor survive far longer than those with 2 or more ${ }^{3,7,16,21}$ Our prospective observational investigation, the JLGK0901 Study, clearly demonstrated that median overall survival after SRS was significantly longer in patients with 1 tumor than in those with 2 to 4 tumors $(13.9$ [95\% CI 12.0-15.6] months vs 10.8 [9.4-12.4] months; HR 0.76 [95\% CI 0.66$0.88], \mathrm{p}=0.0004)$ and those with 5 to 10 tumors $(10.8$ [9.112.7] months; HR 0.78 [95\% CI 0.65-0.96], $\mathrm{p}=0.019) .{ }^{28}$ Recently, Marcus et al. reported that adding cumulative tumor volume to the lung-specific Graded Prognostic Assessment index significantly improved the prognostic value of this index. ${ }^{11}$ Also, Hirshman et al. stated that cumulative intracranial tumor volume offers superior prognostic value relative to the largest intracranial tumor volume for SRStreated BM patients. ${ }^{5}$ Serizawa et al. reported that the postSRS MST of patients with a cumulative tumor volume of $15 \mathrm{~cm}^{3}$ or larger was significantly shorter than that of patients with a cumulative tumor volume less than $15 \mathrm{~cm}^{3} .{ }^{16}$ Therefore, the addition of tumor numbers and cumulative tumor volume as scoring factors to the BSBM system is 
TABLE 2. Univariable and multivariable analyses of survival after SRS in the Yamamoto series

\begin{tabular}{|c|c|c|c|c|}
\hline \multirow[b]{2}{*}{ Variables } & \multicolumn{2}{|c|}{ Univariable } & \multicolumn{2}{|c|}{ Multivariable } \\
\hline & $\mathrm{HR}(95 \% \mathrm{Cl})$ & $p$ Value & $\mathrm{HR}(95 \% \mathrm{Cl})$ & $\mathrm{p}$ Value \\
\hline Sex (male vs female) & $1.416(1.275-1.573)$ & $<0.0001$ & $1.417(1.275-1.577)$ & $<0.0001$ \\
\hline \multicolumn{5}{|l|}{ BSBM } \\
\hline 2 vs 3 & $1.801(1.542-2.111)$ & $<0.0001$ & $1.220(0.600-2.382)$ & 0.5831 \\
\hline 1 vs 2 & $1.772(1.578-1.991)$ & $<0.0001$ & $1.314(0.655-2.543)$ & 0.4435 \\
\hline 0 vs 1 & $1.855(1.566-2.187)$ & $<0.0001$ & $1.398(0.690-2.710)$ & 0.3534 \\
\hline KPS score $(\leq 70 \%$ vs $\geq 80 \%)$ & $2.209(1.973-2.468)$ & $<0.0001$ & $1.395(0.718-2.815)$ & 0.3413 \\
\hline No. of tumors ( $\geq 2$ vs 1 ) & $1.487(1.335-1.659)$ & $<0.0001$ & 1.450 (1.299-1.621) & $<0.0001$ \\
\hline \multicolumn{5}{|l|}{ Tumor vol, $\mathrm{cm}^{3}$} \\
\hline Cumulative ( $\geq 15$ vs <15) & $1.640(1.447-1.854)$ & $<0.0001$ & $1.311(1.078-1.593)$ & 0.0067 \\
\hline Largest tumor $(\geq 10$ vs $<10)$ & $1.427(1.260-1.612)$ & $<0.0001$ & $1.005(0.833-1.209)$ & 0.9606 \\
\hline \multicolumn{5}{|l|}{ Dose, Gy } \\
\hline Minimum (<20 vs $\geq 20)$ & $1.340(1.237-1.579)$ & $<0.0001$ & $1.223(0.976-1.288)$ & 0.1040 \\
\hline Maximum (<35 vs $\geq 35)$ & $1.046(1.863-4.289)$ & 0.3771 & & \\
\hline \multicolumn{5}{|l|}{ Original tumor } \\
\hline Nonlung vs lung & $1.022(0.916-1.140)$ & 0.6911 & & \\
\hline Non- vs well-controlled & $2.400(2.123-2.693)$ & $<0.0001$ & $1.665(0.867-3.333)$ & 0.1320 \\
\hline Extracranial metastases & $1.410(1.275-1.559)$ & $<0.0001$ & $1.078(0.558-2.163)$ & 0.8310 \\
\hline Neurological symptom(s) (yes vs no) & $1.150(1.042-1.270)$ & 0.0056 & $1.105(0.775-1.291)$ & 0.0690 \\
\hline Prior WBRT (yes vs no) & $0.937(0.735-1.218)$ & 0.6146 & & \\
\hline Prior surgery (yes vs no) & $1.300(1.133-1.450)$ & 0.0002 & $1.388(1.202-1.610)$ & $<0.0001$ \\
\hline
\end{tabular}

regarded as significantly improving the prognostic value of this index.

In daily clinical practice, ascertaining tumor volume rather than tumor diameter can be rather difficult, which is obviously a weakness of the present study. In such cases, the convenient formula, tumor volume $=\mathrm{abc} / 2$, where $\mathrm{a}$, $\mathrm{b}$, and $\mathrm{c}$ represent the three-dimensional diameters of the tumor, facilitates estimating tumor volume. The heterogeneity of clinical factors is obviously the major weakness of a retrospective study. Also, our databases have inher- ent patient selection and observational biases. In general, Japanese neurosurgeons are less aggressive in performing surgical removal in elderly than in nonelderly patients. Therefore, the proportions of patients who had undergone surgical removal before SRS were relatively small in these 2 series (16\% in the Y-series and 11\% in the S-series). Furthermore, the major problem associated with WBRT, neurocognitive function decline in elderly patients, has been widely recognized by physicians in Japan managing cancer patients since the 1990s. Therefore, there has been a
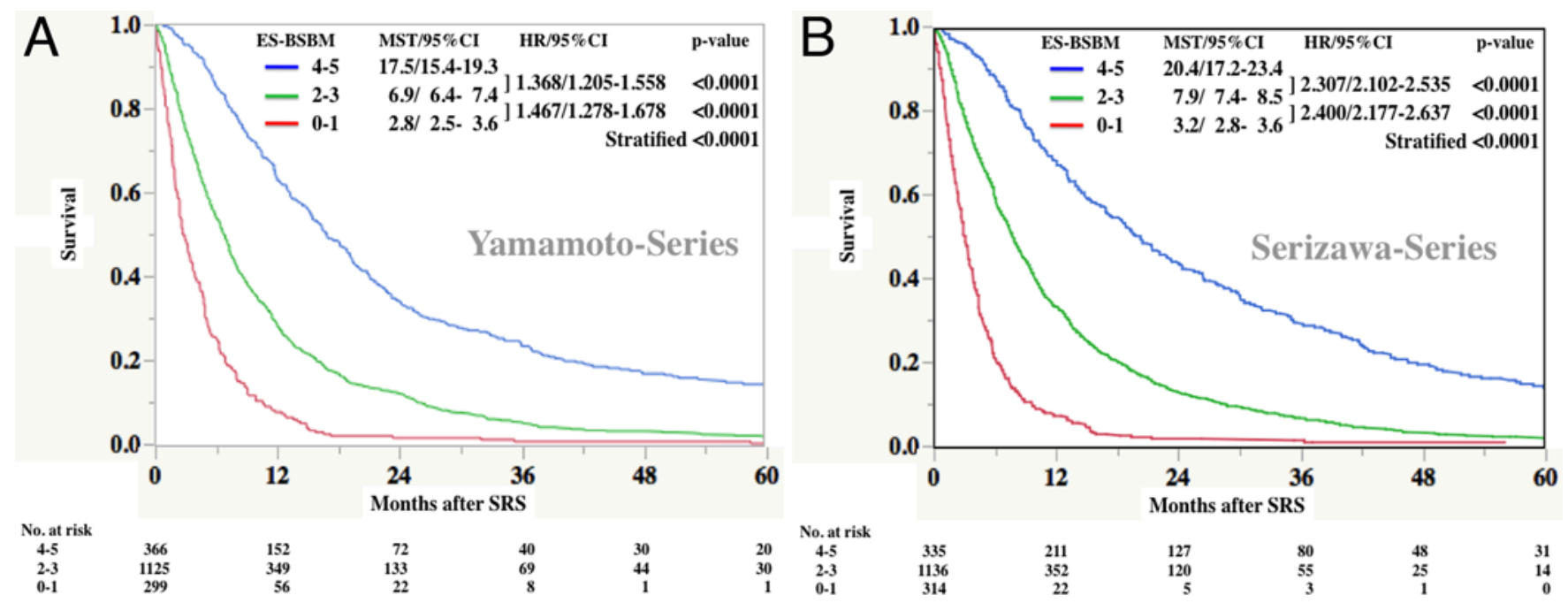

FIG. 2. Kaplan-Meier analysis of overall survival according to ES-BSBM category based on the Y-series (A) and S-series (B) patients. 

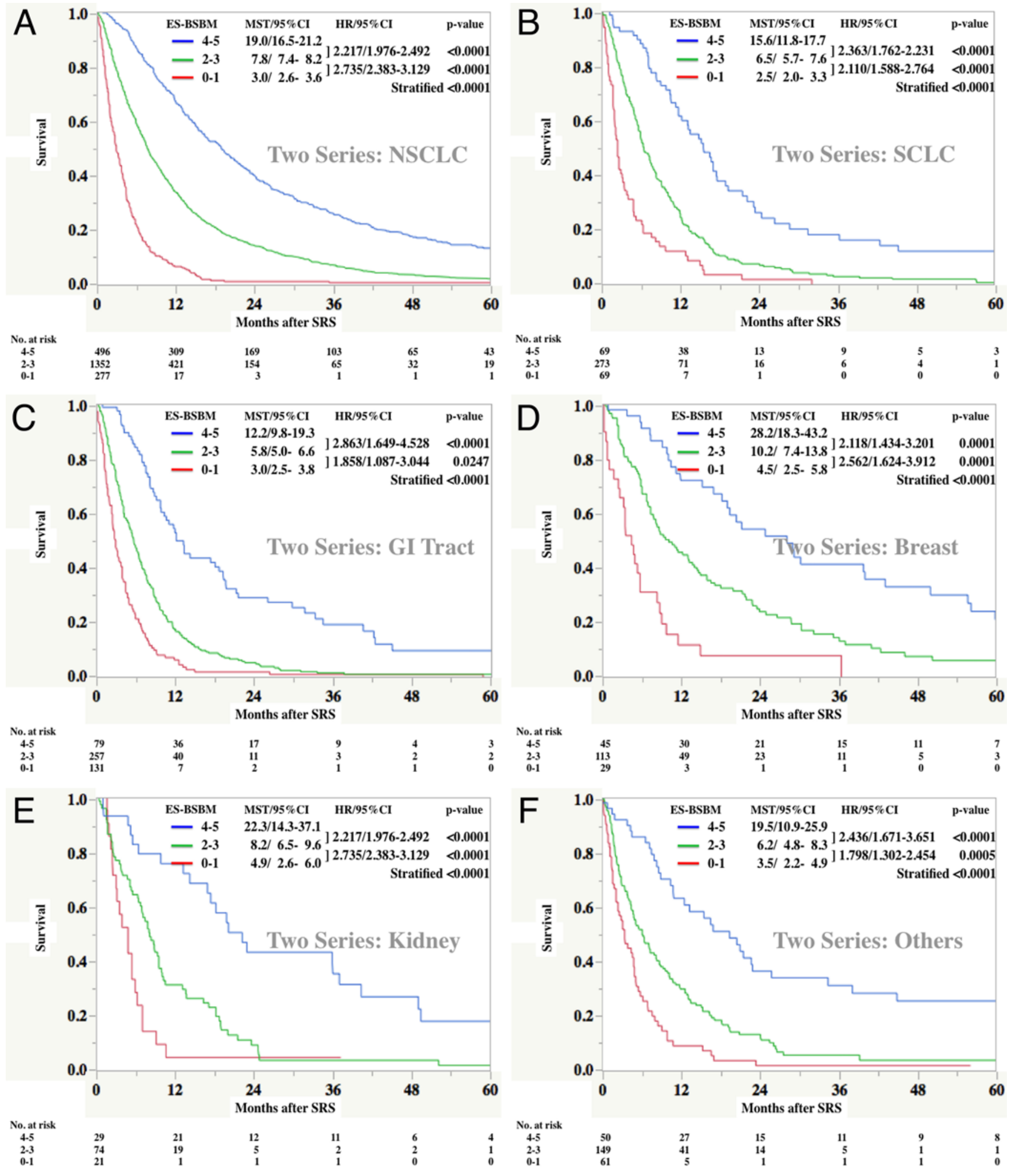

FIG. 3. Kaplan-Meier analysis of overall survival according to the ES-BSBM category based on all patients in the 2 series with respect to NSCLC $(\mathbf{A})$, SCLC $(B)$, gastrointestinal $(\mathrm{GI})$ tract cancer $(\mathbf{C})$, breast cancer $(\mathbf{D})$, kidney cancer $(\mathrm{E})$, and others $(\mathbf{F})$. 

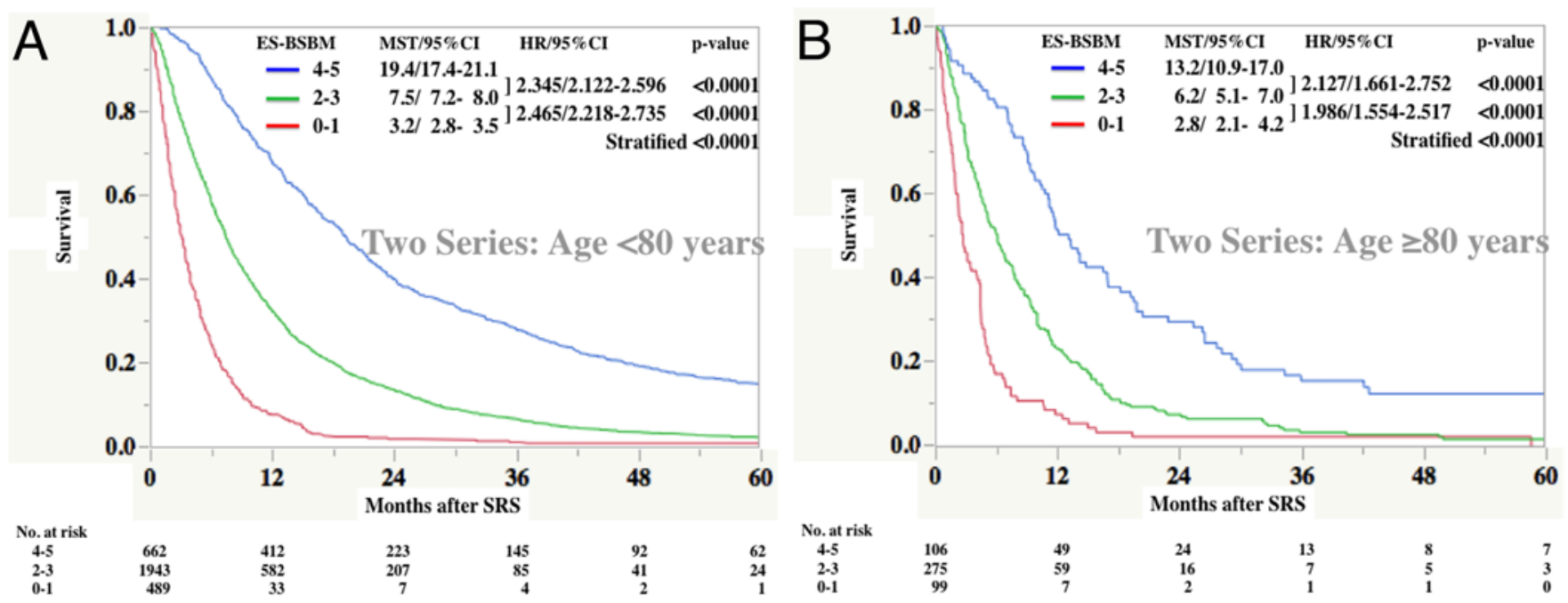

FIG. 4. Kaplan-Meier analysis of overall survival according to the ES-BSBM based on 2 patient age groups: 65-79 years old (A) and 80 years of age or older (B).

tendency for considerable numbers of physicians to favor avoiding WBRT when treating elderly BM patients. The numbers of patients who had undergone pre-SRS WBRT were thus extremely small in the 2 series $(4 \%$ in the Yseries and 6\% in the $\mathrm{S}$-series). These are important issues that must be considered when we use observational data to assess the effects of various treatments and exposures on outcomes. Using the prospective cohort study design is a reasonable approach to minimizing, if not eliminating, the effects of treatment selection bias and confounders.

\section{Conclusions}

In our experience, adding the number of tumors and cumulative tumor volume as scoring factors to the BSBM system significantly improved the prognostic value of this index. The present study validated the ES-BSBM by testing it in a different patient group. The ES-BSBM system was shown to be applicable to patients with all primary tumors as well as those 80 years or older.

\section{Acknowledgments}

We are very grateful to Bierta E. Barfod, MD, Katsuta Hospital

\section{TABLE 3. Outline of ES-BSBM}

\begin{tabular}{clc}
\hline & \multicolumn{2}{c}{ ES-BSBM Scoring Criteria } \\
\cline { 2 - 3 } & \multicolumn{1}{c}{0} & 1 \\
\hline KPS score & $50-70 \%$ & $80-100 \%$ \\
\hline Control of primary tumor & No & Yes \\
\hline Extracranial metastases & Yes & No \\
\hline No. of tumors & $\geq 2$ & 1 \\
\hline Cumulative tumor vol, $\mathrm{cm}^{3}$ & $\geq 15$ & $<15$ \\
\hline Scores & \multicolumn{2}{c}{ ES-BSBM Category } \\
\hline $4 \& 5$ & \multicolumn{2}{c}{$4-5$} \\
\hline $2 \& 3$ & \multicolumn{2}{c}{$2-3$} \\
\hline $0 \& 1$ & \multicolumn{2}{|c}{$0-1$} \\
\hline
\end{tabular}

Mito GammaHouse, for her help in preparing this English manuscript.

\section{References}

1. Chen L, Shen C, Redmond KJ, Page BR, Kummerlowe M, Mcnutt T, et al: Use of stereotactic radiosurgery in elderly and very elderly patients with brain metastases to limit toxicity associated with whole brain radiation therapy. Int J Radiat Oncol Biol Phys 98:939-947, 2017

2. Gaspar L, Scott C, Rotman M, Asbell S, Phillips T, Wasserman T, et al: Recursive partitioning analysis (RPA) of prognostic factors in three Radiation Therapy Oncology Group (RTOG) brain metastases trials. Int J Radiat Oncol Biol Phys 37:745-751, 1997

3. Hanssens P, Karlsson B, Yeo TT, Chou N, Beute G: Detection of brain micrometastases by high-resolution stereotactic magnetic resonance imaging and its impact on the timing of and risk for distant recurrences. J Neurosurg 115:499-504, 2011

4. Higuchi Y, Serizawa T, Nagano O, Matsuda S, Ono J, Sato M, et al: Three-staged stereotactic radiotherapy without whole brain irradiation for large metastatic brain tumors. Int J Radiat Oncol Biol Phys 74:1543-1548, 2009

5. Hirshman BR, Wilson B, Ali MA, Proudfoot JA, Koiso T, Nagano O, et al: Superior prognostic value of cumulative intracranial tumor volume relative to largest intracranial tumor volume stereotactic radiosurgery-treated brain metastasis patients. Neurosurgery 82:473-480, 2018

6. Hotta T, Kohno H, Taniguchi E, Magaki T, Hidaka T, Nishimoto T: Treatment option for elderly patients with metastatic brain tumors. Jpn J Cancer Clin 45:1143-1148, 1999

7. Karlsson B, Hanssens P, Wolff R, Söderman M, Lindquist C, Beute G: Thirty years' experience with Gamma Knife surgery for metastases to the brain. J Neurosurg 111:449-457, 2009

8. Kim SH, Weil RJ, Chao ST, Toms SA, Angelov L, Vogelbaum MA, et al: Stereotactic radiosurgical treatment of brain metastases in older patients. Cancer 113:834-840, 2008

9. Lorenzoni J, Devriendt D, Massager N, David P, Ruíz S, Vanderlinden B, et al: Radiosurgery for treatment of brain metastases: estimation of patient eligibility using three stratification systems. Int J Radiat Oncol Biol Phys 60:218-224, 2004

10. Lutterbach J, Bartelt S, Momm F, Becker G, Frommhold H, 
Ostertag C: Is older age associated with a worse prognosis due to different patterns of care? A long-term study of 1346 patients with glioblastomas or brain metastases. Cancer 103:1234-1244, 2005

11. Marcus LP, Marshall D, Hirshman BR, McCutcheon BA, Gonda DD, Koiso T, et al: Cumulative intracranial tumor volume (CITV) enhances the prognostic value of the lungspecific graded prognostic assessment (GPA) model. Neurosurgery 79:246-252, 2016

12. Minniti G, Esposito V, Clarke E, Scaringi C, Bozzao A, Lanzetta G, et al: Stereotactic radiosurgery in elderly patients with brain metastases. J Neurooncol 111:319-325, 2013

13. Niwińska A, Murawska M: New breast cancer recursive partitioning analysis prognostic index in patients with newly diagnosed brain metastases. Int J Radiat Oncol Biol Phys 82:2065-2071, 2012

14. Noel G, Bollet MA, Noel S, Feuvret L, Boisserie G, Tep B, et al: Linac stereotactic radiosurgery: an effective and safe treatment for elderly patients with brain metastases. Int J Radiat Oncol Biol Phys 63:1555-1561, 2005

15. Rades D, Pluemer A, Veninga T, Schild SE: Comparison of different treatment approaches for one to two brain metastases in elderly patients. Strahlenther Onkol 184:565-571, 2008

16. Serizawa T, Yamamoto M, Sato Y, Higuchi Y, Nagano O, Kawabe T, et al: Gamma Knife surgery as sole treatment for multiple brain metastases: 2-center retrospective review of 1508 cases meeting the inclusion criteria of the JLGK0901 multi-institutional prospective study. J Neurosurg 113 (Suppl):48-52, 2010

17. Sperduto PW, Berkey B, Gaspar LE, Mehta M, Curran W: A new prognostic index and comparison to three other indices for patients with brain metastases: an analysis of 1,960 patients in the RTOG database. Int J Radiat Oncol Biol Phys 70:510-514, 2008

18. Sperduto PW, Chao ST, Sneed PK, Luo X, Suh J, Roberge $\mathrm{D}$, et al: Diagnosis-specific prognostic factors, indexes, and treatment outcomes for patients with newly diagnosed brain metastases: a multi-institutional analysis of 4,259 patients. Int J Radiat Oncol Biol Phys 77:655-661, 2010

19. Sperduto PW, Kased N, Roberge D, Chao ST, Shanley R, Luo $X$, et al: The effect of tumor subtype on the time from primary diagnosis to development of brain metastases and survival in patients with breast cancer. J Neurooncol 112:467-472, 2013

20. Sperduto PW, Yang TJ, Beal K, Pan H, Brown PD, Bangdiwala A, et al: Estimating survival in patients with lung cancer and brain metastases: an update of the graded prognostic assessment for lung cancer using molecular markers (LungmolGPA). JAMA Oncol 3:827-831, 2017

21. Watanabe S, Yamamoto M, Sato Y, Kawabe T, Higuchi Y, Kasuya H, et al: Stereotactic radiosurgery for brain metastases: a case-matched study comparing treatment results for patients 80 years of age or older versus patients 65-79 years of age. J Neurosurg 121:1148-1157, 2014

22. Weltman E, Salvajoli JV, Brandt RA, de Morais Hanriot R, Prisco FE, Cruz JC, et al: Radiosurgery for brain metastases: a score index for predicting prognosis. Int J Radiat Oncol Biol Phys 46:1155-1161, 2000

23. Yamamoto M, Kawabe T, Higuchi Y, Sato Y, Barfod BE,
Kasuya H, et al: Validity of three recently proposed prognostic grading indexes for breast cancer patients with radiosurgically treated brain metastases. Int J Radiat Oncol Biol Phys 84:1110-1115, 2012

24. Yamamoto M, Kawabe T, Sato Y, Higuchi Y, Nariai T, Watanabe $S$, et al: Stereotactic radiosurgery for patients with multiple brain metastases: a case-matched study comparing treatment results for patients with 2-9 versus 10 or more tumors. J Neurosurg 121 (Suppl):16-25, 2014

25. Yamamoto M, Sato Y, Serizawa T, Kawabe T, Higuchi Y, Nagano O, et al: Subclassification of recursive partitioning analysis Class II patients with brain metastases treated radiosurgically. Int J Radiat Oncol Biol Phys 83:1399-1405, 2012

26. Yamamoto M, Serizawa T, Sato Y, Kawabe T, Higuchi Y, Nagano O, et al: Validity of two recently-proposed prognostic grading indices for lung, gastro-intestinal, breast and renal cell cancer patients with radiosurgically-treated brain metastases. J Neurooncol 111:327-335, 2013

27. Yamamoto M, Serizawa T, Shuto T, Akabane A, Higuchi Y, Kawagishi J, et al: Stereotactic radiosurgery for patients with multiple brain metastases (JLGK0901): a multi-institutional prospective observational study. Lancet Oncol 15:387-395, 2014

28. Yancik R, Ries LAG: Cancer in older persons: an international issue in an aging world. Semin Oncol 31:128-136, 2004

29. Yomo S, Hayashi M: Is upfront stereotactic radiosurgery a rational treatment option for very elderly patients with brain metastases? A retrospective analysis of 106 consecutive patients age 80 years and older. BMC Cancer 16:948, 2016

\section{Disclosures}

The authors report no conflict of interest concerning the materials or methods used in this study or the findings specified in this paper.

\section{Author Contributions}

Conception and design: Yamamoto, Serizawa. Acquisition of data: Serizawa, Higuchi, Nagano, Aiyama, Koiso, Watanabe, Kawabe. Analysis and interpretation of data: Yamamoto, Serizawa. Drafting the article: Yamamoto. Critically revising the article: Yamamoto, Serizawa. Reviewed submitted version of manuscript: Yamamoto, Higuchi, Nagano, Aiyama, Koiso, Watanabe, Kawabe. Approved the final version of the manuscript on behalf of all authors: Yamamoto. Statistical analysis: Higuchi, Sato. Study supervision: Yamamoto, Serizawa, Kasuya.

\section{Supplemental Information \\ Previous Presentations}

This work was presented in oral form at the 19th Biannual Congress of the International Leksell Gamma Knife Radiosurgery Society, Dubai, United Arab Emirates, March 4-8, 2018.

\section{Correspondence}

Masaaki Yamamoto: Katsuta Hospital Mito GammaHouse, Ibaraki, Japan.bcd06275@nifty.com. 